\title{
A Case Report on Mild Left Para-Central Bulge of Intervertebral Disc- C4/5 and C5/6 with Mild Narrowing of Left Intervertebral Foramen Inducing Severe Neck Pain and Ipsilateral Symptoms
}

\author{
Muthukumar Arumugam ${ }^{1, *}$, Rumana Khatija', Madhura Bose', Syed Mohasin Abbas', Mohan Alex², Vijayperumal Murugesh ${ }^{2}$ \\ 'Department of Pharmacology, Krupanidhi College of Pharmacy, Bangalore, Karnataka, INDIA. \\ ${ }^{2}$ Shri Kannan Physiotherapy Hospital, Tiruppur, Tamil Nadu, INDIA.
}

\begin{abstract}
Cervical disc bulge or other cervical spine diseases often results into neck and arm pain in patients formulating an etiology of direct impingement of nerve roots and associated inflammatory process. The clinical presentation also corresponds with the ipsilateral symptoms associated with the cervical disorder as depicted in the patient. A 33 year's old South Indian male is diagnosed with Para-central bulge at the level of C4/5 and C5/C6-Intervertebral disc presented to the physical therapy with neck pain radiating into left sided upper and lower extremity pain. A magnetic resonance imaging revealed mild Para central bulge of $\mathrm{C} 4 / 5$ and $\mathrm{C} 5 / 6$-Intervertebral disc with mild narrowing of the left intervertebral foramen. Results from MRI scan show the alignment and the morphology of the vertebrae to be normal. Also, the CSF displays normal intensity in all sequences. The patient reported to physical therapy for cervical pain and Radiculopathy. After evaluation
\end{abstract}

he was asked to continue with regular home exercise programme like cervical lateral flexion stretch, unilateral wall stretch which resulted to reduce the cervical pain and shows improvement in its subsequent symptoms. Key words: Cervical bulge, Intervertebral disc C4/5 and C5/6, Ipsilateral symptoms, Radiculopathy, Medical Representative, Severe Neck Pain.

\section{Correspondence}

Dr. Muthu Kumar A

Department of Pharmacology, Krupanidhi College of Pharmacy, Bangalore560035, Karnataka, INDIA.

Phone: +91-9976696000

Email: mkpharmacologist@gmail.com

DOI: 10.5530/jyp.2020.12.20

\section{INTRODUCTION}

Neck pain is most frequently seen symptoms in clinical studies and is commonly attributed to cervical disc disease. Cervical spine disease is commonly seen in adults and reaches a prevalence of nearly $95 \%$ by the age of 65 years old. Despite various biological factors that have been related to disc diseases, abnormal spinal motion and consequent abnormal loading have been assumed to be the etiology of spinal pathology. ${ }^{1-3}$ Nowadays even children especially students face the same issue due to overloaded school bags or constant straining their neck muscles while reading in same position for hours. Many professionals handling with computer or constant reading may complain of the same, later or sooner due to constant stress with minimal or no exercise at all. This spinal disorder can lead to various disc diseases like instability, neurological deficit, spinal stenosis, facet dysfunction. These clinical symptoms can lead to loss of neck movement and can cause disability to patients. These disc herniation induce various effects depending on amount of disc protrusion. If the disc protrudes enough it may impinge on a spinal nerve as it exits the vertebral column and this impingement may cause radiating symptoms into the peripheral extremities. Cervical disc herniation usually occurs when flexion, extension, rotation and their combination exceeds the strength of annulus fibrosis. With the combination of these movements, it increases the amount of pressure on the disc thus increasing the chance of disc herniation. Cervical disc herniation results from the displacement of nucleus pulposus of the intervertebral disc at the cervical level, which may lead to direct compression of the spinal cord or impingement of the nerve roots. ${ }^{4,5}$ Some of the symptoms includes as ipsilateral pain in the neck, or pain radiating down the ipsilateral arm to fingers. The pain they experience could be dull or sharp. Neck flexion and arm abduction over the highest of the pinnacle might yield an equivalent impact. The ipsilateral nature of the above symptoms is the hallmark of cervical herniation disrupting the nerve roots on the same side. An effect of decreased hydration in the disc is the decreased strength of the annulus fibrosis. As the disc becomes less hydrated, the annulus fibre becomes stiffer and less pliable. This can lead to the cracking of the annulus fibrosis and on adding compression to the disc the nucleus pulposus could protrude out through cracked fibres. ${ }^{6-8}$

\section{CASE PRESENTATION}

A 33 years old south Indian male working as a Medical Representative is diagnosed with mild left Para-central bulge at the level of C4/5 and C5/6-Intervertebral disc with mild narrowing of the left intervertebral foramen. Magnetic Resonance Imaging (MRI) report confirmed this diagnosis. He presented to physical therapy with complaints of neck pain radiating to his left upper and lower extremity were shown in (Figure 1). $\mathrm{He}$ also informed about the tingling sensation in left upper extremity and achy feeling with symptoms radiating to his left side of the body were shown in (Figure 2). He complained about the pain getting worse during sitting and supine positions were observed in (Figure 3,4). The pain was alleviated with walking. He also informed to suffer from pain from past 6 months and notified the cause to be excessive workload, constant driving demand at his job, lack of rest and spending excess time in computer with irregular diet and exercise routine. For subject denied from any past medical and surgical history. His family history was unremarkable except for myocardial infarction. 
He denied the use of alcohol, cigarettes, or any recreational drugs. MRI reports suggested no significant abnormality to be seen in dorsal and lumbar spine and a general review to be all normal. MRI examination of the cervical spine was performed in the sagittal and axial plane using T1W, T2W and short T1-inversion recovery (STIR). MRI scan shows a loss of disc height and bulging at $\mathrm{C} 4 / 5$ and C5/6 level. All alternative cervical levels were traditional while not central canal pathology or neural foraminal pathology. The alignment of the vertebrae is normal. The vertebral bodies, pedicles, laminae, transverse processes shows normal morphology MR signal pattern. The facet joint also appears normal. The cervical canal AP dimensions from $\mathrm{C} 2$ to $\mathrm{C} 7$ were shown in (Table 1). The scan report reveals that cervical spinal cord and the CSF display normal signal intensity in all sequences. The cranio vertebral junction is normal. The atlanto-axial joint is normal. There is no evidence of tonsilar herniation. The pre and Para-spinal regions do not show any abnormal signal intensity. Also, no significant abnormality is seen in dorsal and lumbar spine. This study was approved by the Institutional Ethical Committee (IEC No: PGP-12/25/10/19).

\section{DISCUSSION}

Cervical disc herniation is a condition that is seldom of a single treatment event. It is a progressive disorder that usually presents as pain and weakness within the neck and arm involving ipsilateral diverging pain that corresponds to cervical levels affected. ${ }^{9-11}$ Many times

Table 1: MRI Report - Cervical Spine with Whole Spine Screening.

\begin{tabular}{ccccccc}
\hline Level & C2-C3 & C3-C4 & C4-C5 & C5-C6 & C6-C7 & C7-D1 \\
\hline Cm & 1.27 & 1.10 & 1.04 & 1.05 & 0.94 & 1.04 \\
\hline
\end{tabular}

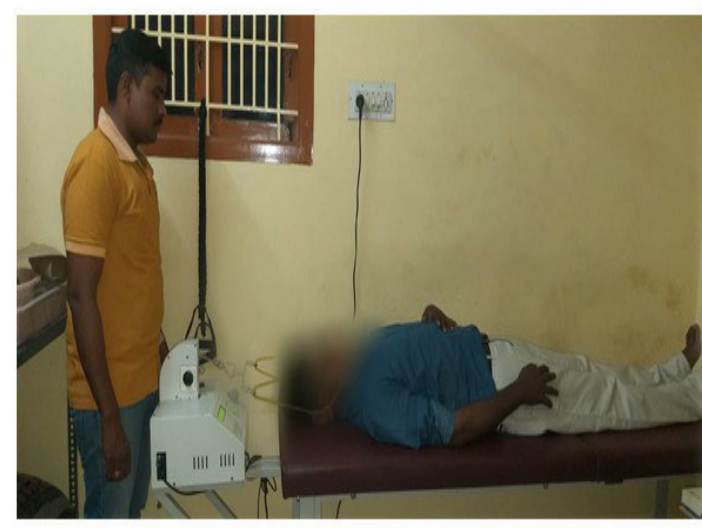

Figure 1: Neck Pain Radiating to Left Upper and Lower Extremity.

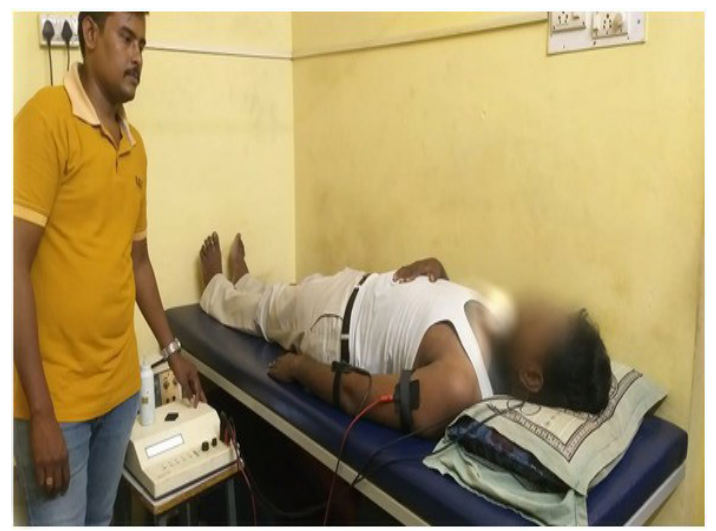

Figure 2: Achy Feeling with Symptoms Radiating to Left Side of the Body. surgery has been used as a final resort treatment for cervical pathology. Conservative treatment is more widely used to treat cervical dysfunction and is generally believed to alleviate symptoms of cervical radiculopathy. Our patient's presentation depicts the mild Para-central bulging at the level of C4/5 and C5/6- Intervertebral disc with mild narrowing of left intervertebral foramen that resulted into pain radiating into upper and lower extremity showing ipsilateral symptoms. The herniation depicts cervical radiculopathy by affecting the nerve roots at the spinal level, but the symptoms on the contralateral side can be due to impingement of the nerve root at the spinal level. Such cases can result into a disorder as Brown-Sequard syndrome depicting ipsilateral weakness and contralateral loss of pain and temperature which are the principle findings. ${ }^{12-15}$ An explanation of our patient's presentation requires consideration of the anatomy of the spinal cord. The spinothalamic tract that could be an anterolateral system or the ventrolateral system is a sensory pathway from the skin right to the neural structure. From the ventral posterolateral nucleus in the thalamus, sensory information is relayed upwards to the somatosensory cortex of the post central gyrus. ${ }^{16-18}$ This tract mainly plays a role in pain and temperature signal transmission. When a stimulus is elicited, the signal enters the dorsal horn of the corresponding spinal level and synapses with the secondary neuron. The secondary cell crosses to the choice side via the anterior white commissure and ascends until it terminates inside the ventral posterolateral nucleus, ventral posterior oralis nucleus, ventral posterior inferior nucleus and posterior a part of the ventral medial nucleus of the neural structure. The lateral spinothalamic tract shows greater clinical importance than its anterior counterparts as it transmits impulses concerned with pain. Here we propose the possibility that this tract was disrupted by the C5/6 bulge and resulted in our patient's symptomatology. The ipsilateral leg pain reported by our subject is most likely due to the direct compression of the ipsilateral spinothalamic tract, which crosses over at the lumbrosacral levels. ${ }^{19-21}$ The physician guided
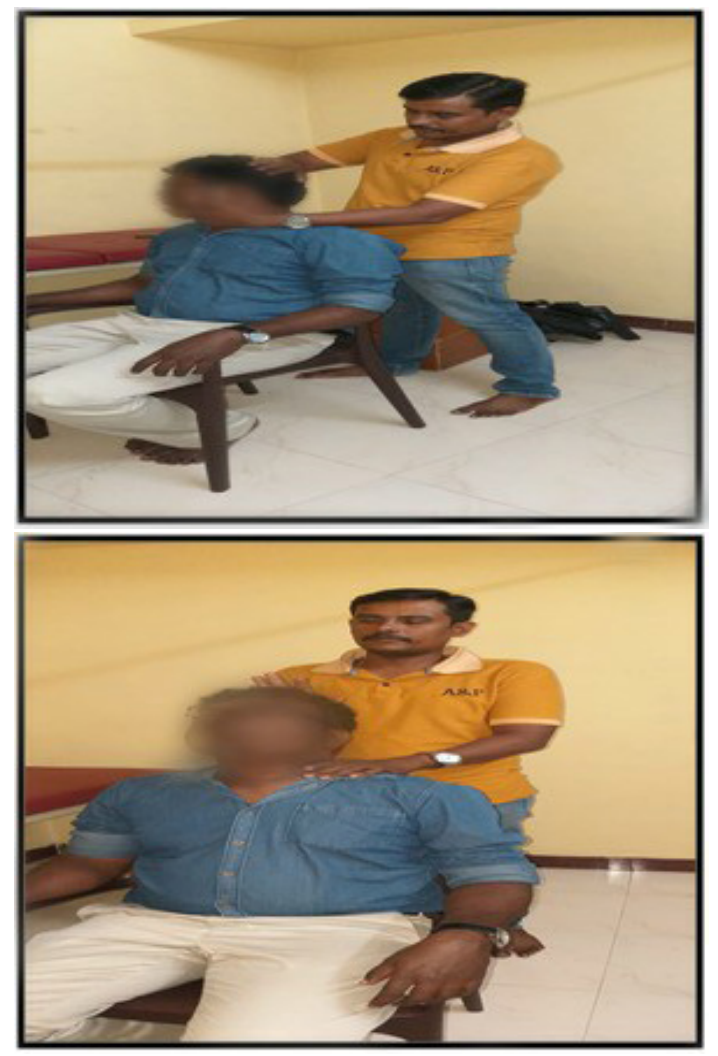

Figure 3, 4: Pain Getting Worse During Sitting and Supine Positions. 
our subject to take adequate amount of rest with minimal stress-related activities and suggested to implement Cervical Traction for Disc Bulge and Interferential Therapy for radiating muscle pain with Cervical Collar Application if required to do so with some basic muscle strengthening exercises. He also mentioned about Ultrasound Therapy for effective results. Our subject did not undergo any surgery or cervical traction methodology, instead opted for some home exercises as suggested by his physiotherapist. During the follow-up he informed to notice some minor improvements which he believed on continuation can show great results. Our subject also mentioned the use of ayurveda therapy for given period of time with some basic muscular exercise that have shown noticeable improvement in reduction of the radiating ipsilateral pain in the legs and arms. He shared about his improvement in his diet mentioning reduce intake of cholesterol containing items or junk foods and following timely visit to physiotherapist have helped him to cope with his pain and performing daily activities. ${ }^{22}$

\section{CONCLUSION}

Our case report depicts our patient's experience with severe neck pain which radiates ipsilaterally showing muscular pain in his leg and arms. No alternative focal medicine deficits were found, apart from bilateral hyperreflexia and twitch of the lower limbs. MRI scans revealed mild left para-central bulge of intervertebral disc- C4/5 and C5/6 with mild narrowing of left intervertebral foramen. It is postulated that the lateral spinothalamic tract could be involved due to cord compression by the bulge in the disc, resulting in predominantly ipsilateral symptoms. An intervention to the resolution was the combination of a home exercise programme with Ayurveda Therapy has shown great results and improvement. Maintenance of the diet with scheduled visit to physiotherapist can lead to complete resolution of his symptoms. It is postulated that the lateral spinothalamic tract could be involved due to cord compression by the bulge in the disc, resulting into ipsilateral symptoms. Our subject's case may provide further insights into other atypical presentations in the cervical disc bulge at $\mathrm{C} 4 / 5$ and $\mathrm{C} 5 / 6$ level.

\section{MANAGEMENT}

Our subject's physiotherapists have suggested him with home exercise with some muscle strengthening activities to be followed on regular basis for better results with the Ayurveda Therapy. The main aim to focus on muscle strengthening is to improve the tone and flexibility of the peripheral spine muscles surrounding the bulge that may help to reduce the direct pressure on the affected zone and can reduce the impingement of the nerve at the spinal level resulting into radiation of the ipsilateral pain. Working with resistance bands, climbing stairs, cycling, indulged into fun activity like dancing or aerobics, heavy exercises like push ups, squats or weight lifting can help strengthening of the spinal muscles. Better results can be achieved with proper diet including protein based food like Eggs, Salmon fish, Chicken breast, Yogurt, Shrimp and Prawn, Soybean, Dairy products. Avoiding junk food and Cholesterol rich food must be the first priority of the patient to achieve better results.

\section{ACKNOWLEDGEMENT}

We take this opportunity to express our gratitude and respectful thanks to Dr. Suresh Nagpal, Chairman, Krupanidhi Group of Educational Institutions Bangalore. I would like extend my respectful thanks to Dr.
Amitkumar Das, Principal, Krupanidhi College of Pharmacy and Bangalore.

\section{AUTHOR CONTRIBUTION}

All authors are having equal contribution.

\section{CONFLICT OF INTEREST}

The authors declare no conflict of interest.

\section{REFERENCES}

1. Yeung JT, Johnson JI, Karim AS. Cervical disc herniation presenting with neck pain and contralateral symptoms: A case report. Journal of Medical Case Reports. 2012;6(1):166.

2. Weishaupt $D$, Zanetti M, Hodler J, et al. MR imaging of the lumbar spine: Prevalence of intervertebral disk extrusion and sequestration, nerve root compression, end plate abnormalities and osteoarthritis of the facet joints in asymptomatic volunteers. Radiology. 1998;209(3):661-6.

3. Baptiste DC, Fehlings MG. Pathophysiology of cervical myelopathy. Spine J. 2006;6(6):190-7.

4. Sayer FT, Vitali AM, et al. Brown-Sequard syndrome produced by C3-C4 cervical disc herniation: A case report and review of the literature. Spinal Cord. 2008;33(9):279-82.

5. Lee JK, Kim YS, Kim SH. Brown-Sequard syndrome produced by cervical disc herniation with complete neurologic recovery: Report of three cases and review of the literature. Spinal Cord. 2007;45(11):744-8.

6. Craig AD. Retrograde analyses of spinothalamic projections in the macaque monkey: Input to ventral posterior nuclei. J Comp Neurol. 2006;499(6):965-78.

7. Sherry T, Peter P. The management of a patient with a cervical disc herniation: A case report. Clinical Medicine: Case Reports. 2008;1:45-9.

8. Callaghan JP, McGill SM. Intervertebral disc herniation: Studies on a porcine model exposed to highly repetitive flexion/extension motion with Compressive force. Clin Biomech. 2001;16(1):28-37.

9. Andrest WJ, Baillargeon $\mathrm{E}$, et al. Validation of A Non-Invasive Technique to Precisely Measure the in-vivo three-dimensional cervical spine movement. Spine. 2011;36(6):393-400.

10. Costi JJ, Hearn TC, et al. The effect of hydration on the stiffness of intervertebral disc in an ovine model. Clin Biomech. 2002;17(6):446-55.

11. Goel VK, Clausen JD. Prediction of Load Sharing Among Spinal Components of a C5C6 Motion Segment Using the Finite Element Approach. Spine. 1998;23(6):684-91.

12. Chung TS, LeeYJ, et al. Reducibility of cervical disc herniation: Evaluation at MR imaging during cervical traction with nonmagnetic traction device. Radiology. 2002;225(3):895-900

13. Norkin CC, White DJ. Measurement of joint motion. Philadelphia, PA: FA. Davis Company. 1985;2:181-98

14. Aour B, Damba N. Finite Element Investigation of the Intervertebral Disc Behaviour, Comput. Methods Biomech Biomed Eng. 2014;17:58-9

15. Stemper BD, Yoganandan N, et al. Gender Dependent CervicaL Spine Segmental Kinematics during Whiplash. J Biomech. 2003;36(9):1281-9.

16. Li G, DeFratel LE, Park SE, et al. In-vivo Articular Cartilage Contact Kinematics of the Knee: An investigational using Dual-Orthogonal Fluoroscopy and Magnetic Resonance Image Based Computer Models. Am J Spoets Med. 2005;33(1):102-7.

17. Malcolm GP. Surgical Disorder of the Cervical Spine: Presentation and Management of Common Disorders. J Neurol Neurosurg Psychiatry. 2002;73(Suppl 1):34-41.

18. Stemper BD, Yoganandan N, Pintar FA. Gender Dependent Cervical Spine Segmental Kinematics During Whiplasxh. J Biomech. 2003;36(9):1281-9.

19. Hunter $L Y$, Braunstein EM, et al. Radiographic changes following Anterior Cervical Fusion. Spine. 1980;5(5):399-401.

20. Deng B, Begeman PC, et al. Kinematics of Human Cadaver Cervical Spine dur ing Low Speed Rear-end impacts. Stapp Car Crash J. 2000;44:171-88.

21. Barsa $P$, Suchomel $P$. Factors affecting saggittal malalignment due to cage subsidence in standalone cage assisted anterior cervical fusion. Eur Spine J 2007;16(9):1395-400.

22. Wang S, Xia Q, et al. Measurement of geometric deformation of lumbar interver tebral discs under in-vivo weight-bearing condition. Biomech. 2011;42(6):705-11.

\footnotetext{
Article History: Submission Date :13-01-2020 ; Revised Date : 28-01-2020 ; Acceptance Date : 02-02-2020

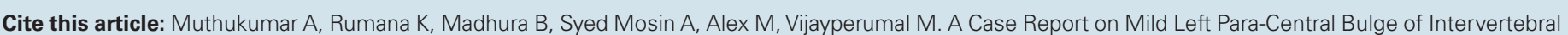

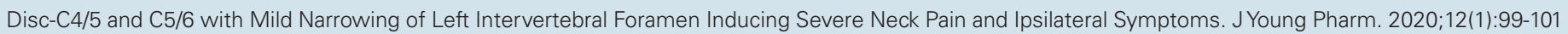

